\title{
LIFTING INVERTIBLES IN VON NEUMANN ALGEBRAS
}

\author{
ROBERT R. ROGERS
}

(Communicated by Palle E. T. Jorgensen)

\begin{abstract}
Given $\mathscr{B}(\mathscr{H})$, the algebra of bounded operators on a separable Hilbert space $\mathscr{H}$, and $\mathscr{K}$, the ideal of compact operators, it is a well-known fact that $T$ in $\mathscr{B}(\mathscr{H})$ is a Fredholm operator if and only if $\pi(T)$ is invertible in $\mathscr{B}(\mathscr{H})$ where $\pi$ is the canonical quotient map. A natural question arises: When can a Fredholm operator be perturbed by a compact operator to obtain an invertible operator? Equivalently, when does the invertible element $\pi(T)$ lift to an invertible operator? The answer is well known: $T$ may be perturbed by a compact operator to obtain an invertible operator if and only if the Fredholm Index of $T$ is 0 . In this case, the perturbation may be made as small in norm as we wish. Using the generalized Fredholm index for a von Neumann algebra developed by C. L. Olsen [3], the following generalization is obtained: let $\mathfrak{A}$ be a von Neumann algebra with norm closed ideal $\mathfrak{F}$ and canonical quotient map $\pi$. Let $T \in \mathfrak{A}$ be such that $\pi(T)$ is invertible. Then there exists $K \in \mathfrak{F}$ such that $T+K$ is invertible if and only if $\operatorname{ind}(T)=0$.
\end{abstract}

\section{BASIC FACTS AND NOTATION}

Throughout this discourse $\mathfrak{A}$ denotes a von Neumann algebra of operators on a Hilbert space $\mathscr{H}$. By a projection in $\mathfrak{A}$ we mean a selfadjoint indempotent. For $A \in \mathfrak{A}$, let $R_{A}$ denote the range projection $A$ and let $N_{A}$ denote the projection onto the kernel of $A$. Note that $R_{A}, N_{A} \in \mathfrak{A}$. Let $E, F$ be projections in $\mathfrak{A}$. Define the relation $E \sim F$ if there is a partial isometry $V \in \mathfrak{A}$ with $V^{*} V=E$ and $V V^{*}=F$. One can show that $\sim$ is an equivalence relation on the set of projections in $\mathfrak{A}$. We write $E \lesssim F$ if there exists a partial isometry $V \in \mathfrak{A}$ with $V^{*} V=E$ and $V V^{*} \leq F$. One can show, (see [4, p. 291]), that $E \lesssim F$ and $F \lesssim E$ implies $E \sim F$.

Definition. A projection $E$ in $\mathfrak{A}$ is finite relative to $\mathfrak{A}$ if $E \sim F \leq E \Rightarrow E=F$. Definition. $A \in \mathfrak{A}$ is finite if $R_{A}$ is finite.

Let $\mathfrak{R}$ denote the norm closed, 2-sided ideal generated by the finite elements of $\mathfrak{A} ; \mathfrak{R}$ is called the relatively compact ideal of $\mathfrak{A}$ and the elements of $\mathfrak{R}$

Received by the editors January 21, 1990.

1980 Mathematics Subject Classification (1985 Revision). Primary 47A53, 47C15; Secondary 46L35.

Key words and phrases. von Neumann Algebra, Fredholm, generalized dimension function, generalized index. 
are called relatively compact. In the case where $\mathfrak{A}=\mathscr{B}(\mathscr{H})$, the algebra of bounded operators on a Hilbert space $\mathscr{H}$, the relatively finite elements are the finite rank elements, and $\mathfrak{R}$ is the actual compact ideal in $\mathscr{B}(\mathscr{H})$.

Let $\mathfrak{I}$ denote an arbitrary norm closed, 2-sided ideal in $\mathfrak{A}$ and let $\pi: \mathfrak{A} \rightarrow$ $\mathfrak{A} / \mathfrak{I}$ denote the canonical quotient map.

Definition. $A \in \mathfrak{A}$ is Fredholm (relative to $\mathfrak{I}$ ) if $\pi(A)$ is invertible in $\mathfrak{A} \mathfrak{I}$.

The following theorem was conceived by $M$. Breuer [1] for the ideal $\mathfrak{R}$ of relatively compact elements and is proven by $\mathrm{C}$. Olsen [3] for general ideals.

Theorem 1. $A \in \mathfrak{A}$ is Fredholm relative to $\mathfrak{I} \Leftrightarrow$ the range of $A$ contains the range of $I-E$ for some projection $E$ in $\mathfrak{I}$, and $N_{A} \in \mathfrak{I}$. In this case $E$ can be chosen so that $\|E A\| \leq \varepsilon$ for any $\varepsilon>0$.

\section{THE DEFINITION OF THE GENERALIZED INDEX FUNCTION}

The following can be found in [3].

Let $\mathfrak{Z}$ be the center $\mathfrak{A}$, with maximum ideal space $\Omega$; identify $\mathfrak{Z}$ with $C(\Omega)$. There is a generalized dimension theory of J. Tomiyama [5], which for each projection $E \in \mathfrak{A}$ gives a continuous cardinal valued dimension function $\operatorname{dim}(E)$ on $\Omega$. The set $\operatorname{dim} \mathfrak{I}$ consisting of all $\operatorname{dim}(E)$ for $E \in \mathfrak{I}$ is called an ideal base and uniquely determines the ideal $\mathfrak{I}$ [6]. For each $A \in \mathfrak{A}$, define the continuous function $i(A)$ by

$$
i(A)=\operatorname{dim} N_{A}-\operatorname{dim} N_{A^{*}}
$$

( $i(A)$ is defined pointwise on the open set where $\operatorname{dim} N_{A} \neq \operatorname{dim} N_{A^{*}}$, extended continuously to its closure, and is zero on the complement of this clopen set). This index map reduces to the usual index map in the classical case and enjoys the properties of the index for a certain class of ideals in $\mathfrak{A}$. For details, see [3, pp. 16-20]. The definitions and notation introduced there are used here.

\section{LIFTING INVERTIBLES}

The problem of lifting invertibles is divided into two cases: (relatively) compact ideals and noncompact ideals. As will be seen, any von Neumann algebra $\mathfrak{A}$ and any closed two sided ideal $\mathfrak{I} \subseteq \mathfrak{A}$ can be divided into two direct summands: on one summand the ideal is compact and on the other the ideal is completely noncompact.

Definition. The ideal $\mathfrak{I}$ is (relatively) compact if $\mathfrak{I} \subseteq \mathfrak{R}$, the ideal of relatively compact elements of $\mathfrak{A}$.

By mimicking the proof of a similar theorem in [2], we answer the question of lifting invertibles in the case where the ideal is compact.

Theorem 2. Let $\mathfrak{I} \subseteq \mathfrak{A}$ be compact, and let $T \in \mathfrak{A}$ be Fredholm relative to $\mathfrak{I}$. Then

$$
\exists K \in \mathfrak{I} \text { with } T+K \text { invertible } \Leftrightarrow i(T)=0 .
$$

In this case, $\|K\|$ can be made as small as we wish. 
Proof. $(\Rightarrow)$ If $T+K$ is invertible then $i(T+K)=0$. But since $K$ is (relatively) compact then $i(T)=i(T+K)=0$ as the index map is invariant under compact perturbations [3, Theorem 7.4].

$(\Leftarrow)$ Suppose $i(T)=0$ and let $\varepsilon>0$. By Theorem 1 , there is a projection $E \in \mathfrak{I}$ with $(I-E)(\mathscr{H}) \subseteq T(\mathscr{H})$ and $\|E T\| \leq \varepsilon$. Thus $T-E T$ has closed range and $i(T-E T)=0$. By [3, Proposition 5.15], $\operatorname{dim} N_{T-E T}=\operatorname{dim} N_{(T-E T)^{*}}$ so there is a partial isometry $U$ with initial space $\operatorname{ker}(T-E T)$ and equal to $(T-E T)(\mathscr{H})^{\perp}$. Thus $K=-E T+\varepsilon U \in \mathfrak{I}$ with $\|K\| \leq 2 \varepsilon$ and it is easily verified that $T+K$ is invertible.

The case where $\mathfrak{I}$ contains some noncompact elements is more complicated as the mapping $i$ is not necessarily invariant under noncompact ideal perturbations. In this case the index map must be modified. Again the reader is referred to $[3, \S 9]$ for details.

Definition. Let $\mathfrak{I}$ be an ideal in a von Neumann algebra $\mathfrak{A}$. Let $\Delta$ denote the following open subset of the maximal ideal space $\Omega$ of $\mathfrak{Z}$ :

$$
\Delta=\left\{t \in \Omega: \exists f \in \operatorname{dim} \mathfrak{I} \text { with } f \geq \aleph_{0} \text { on a neighborhood of } t\right\} .
$$

If $\Delta$ is dense in $\Omega$ for $\mathfrak{I}$, we say that $\mathfrak{I}$ is completely noncompact.

Proposition. For any ideal $\mathfrak{I}$ of $\mathfrak{A}, \exists$ a unique central projection $P$ such that $P \mathfrak{I}$ is completely noncompact in $P \mathfrak{A}$, and $(I-P) \mathfrak{I}$ is relatively compact $(I-P) \mathfrak{A}$.

As a consequence of the above proposition, we see that the Fredholm and index theories for arbitrary $\mathfrak{I}$ split into consideration of the (relatively) compact part of $\mathfrak{I}$ and the completely noncompact part of $\mathfrak{I}$. We assume that $\mathfrak{I}$ is completely noncompact as the compact case has already been considered.

Definition. Let $\mathfrak{I}$ be a completely noncompact ideal in $\mathfrak{A}$. Consider $\mathbb{J} \subseteq C_{C}(\Omega)$ given by

$$
\mathbb{J}=\left\{f \in C_{C}(\Omega):|f| \in \operatorname{dim} \mathfrak{I} \text { and } f \text { is zero in a neighborhood of } \Omega \backslash \Delta\right\} .
$$

We say $f$ is equivalent to $g$ modulo $\mathfrak{I}$ and write $f \equiv g(\bmod \mathbb{J})$ if $f-g \in \mathbb{J}$.

Definition. Let $\mathfrak{I}$ and $\mathbb{J}$ be as above. Define $\bar{i}: \mathfrak{A} \rightarrow C_{C}(\Omega) / \mathbb{J}$ by

$$
\bar{i}(A)=[i(A)], \quad \text { the equivalence class of } i(A) .
$$

Theorem 3. Assume $\mathfrak{I} \subseteq \mathfrak{A}$ is completely noncompact. Let $T \in \mathfrak{A}$ be Fredholm with respect to $\mathfrak{I}$. Then

$\exists K \in \mathfrak{I}$ with $T+K$ invertible $\Leftrightarrow \bar{i}(T)=[0], \quad$ the equivalence class of 0. Proof. $(\Rightarrow)$ If $T+K$ is invertible, then $\bar{i}(T)=\bar{i}(T+K)=[0]$ as $\bar{i}$ is invariant under ideal perturbations. 
$(\Leftarrow)$ Suppose conversely that $\bar{i}(T)=[0]$ (i.e., $i(T)=0$ in a neighborhood of $\Omega \backslash \Delta)$. First choose a projection $E \in \mathfrak{I}$ with $(I-E)(\mathscr{H}) \subseteq T(\mathscr{H})$. Thus $S=T-E T$ has closed range and $\bar{i}(S)=\bar{i}(T)$. We therefore concentrate on $S$.

Let $C$ be a closed and open (clopen) neighborhood of $\Omega \backslash \Delta$ such that $i(S)=$ 0 on $C$. Let $\chi_{C}$ denote the central projection corresponding to the characteristic function of $C$. (Recall that $\mathfrak{Z}$ was identified with $C(\Omega)$ ). Then we have

$$
S=\left[\begin{array}{cc}
\chi_{C} S & 0 \\
0 & \chi_{\Omega \backslash C} S
\end{array}\right] .
$$

Since $S$ has closed range, $\chi_{C} S$ has closed range and $i\left(\chi_{C} S\right)=0$, so by using the argument of the previous theorem we can perturb $\chi_{C} S$ by an element of $\chi_{C} \mathfrak{I}$ to obtain an invertible in $\chi_{C} \mathfrak{A}$. Thus we concentrate on $\chi_{\Omega \backslash C} S$; i.e., we assume that $\Delta=\Omega$.

Lemma. If $\Delta=\Omega$ then $\exists$ a projection $P^{\prime}$ such that $P^{\prime} \in \mathfrak{I}, \operatorname{dim} P^{\prime} \geq \aleph_{0}$, $N_{S} \lesssim P^{\prime}$, and $N_{S^{*}} \lesssim P^{\prime}$.

Proof. For each $t \in \Omega, \exists f_{t} \in \operatorname{dim} \mathfrak{I}$ such that $f_{t} \geq \aleph_{0}$ on a neighborhood $U_{t}$ of $t$. Cover $\Omega$ by finitely many $U_{1}, \ldots, U_{n}$ with corresponding functions $f_{1}, \ldots, f_{n}$. Let $f=\sup \left\{\operatorname{dim} N_{S}, \operatorname{dim} N_{S^{*}}, f_{1}, \ldots, f_{n}\right\}$. Since $\operatorname{dim} \mathfrak{I}$ is an ideal base, $f \in \operatorname{dim} \mathfrak{I}$, and there is a projection $P^{\prime} \in \mathfrak{I}$ with $\operatorname{dim} P^{\prime}=f$. This is the desired $P^{\prime}$, and the lemma is proved.

We now make another reduction: let

$$
X=\left\{t \in \Omega:\left(\operatorname{dim} N_{S}\right)(t)<(\operatorname{dim} I)(t)\right\} .
$$

Thus $\bar{X}$ is clopen and on $\Omega \backslash \bar{X}$ we have $\operatorname{dim} N_{S}=\operatorname{dim} I$. Thus $\chi_{\Omega \backslash \bar{X}} N_{S} \sim$ $\chi_{\Omega \backslash \bar{X}}$ and $\chi_{\Omega \backslash \bar{X}} \in \mathfrak{I}$. So

$$
S=\left[\begin{array}{cc}
\chi_{\bar{X}} S & 0 \\
0 & \chi_{\Omega \backslash \bar{X}} S
\end{array}\right]
$$

may be replaced by

$$
S^{\prime}=\left[\begin{array}{cc}
\chi_{\bar{X}} S & 0 \\
0 & \chi_{\Omega \backslash \bar{X}}
\end{array}\right]=\left[\begin{array}{cc}
\chi_{\bar{X}} S & 0 \\
0 & I
\end{array}\right] .
$$

Note that $S-S^{\prime}=\chi_{\Omega \backslash \bar{X}} S-\chi_{\Omega \backslash \bar{X}} \in \mathfrak{I}$ and $S^{\prime}$ still has closed range. Hence we may concentrate on $\chi_{\bar{X}} S$ and assume that $\operatorname{dim} N_{S}<\operatorname{dim} I$ a.e. on $\Omega$ (i.e., $\bar{X}=\Omega$.

Since $\operatorname{dim} N_{S}+\operatorname{dim}\left(I-N_{S}\right)=\operatorname{dim} I$ and $\operatorname{dim} I \geq \operatorname{dim} P^{\prime} \geq \aleph_{0}, \operatorname{dim}\left(I-N_{S}\right)=$ $\operatorname{dim} I$ a.e. (and hence everywhere) on $\Omega$. Thus $\operatorname{dim}\left(I-N_{S}\right) \geq \operatorname{dim} P^{\prime}$, and so there exists $P \sim P^{\prime}$ such that $P \leq\left(I-N_{S}\right)$. Let $Q^{\prime}=R_{S P}$ and let $Q=Q^{\prime} \oplus N_{S^{*}}$ (note that $Q^{\prime} \perp N_{S^{*}}$ ). 
Note that $Q^{\prime} \sim P$. To see this consider $S P$; clearly $I-P \leq N_{S P}$. To obtain the reverse inequality observe for $x \in \mathscr{H}$

$$
\begin{aligned}
S P x=0 & \Rightarrow P x \in N_{S}(\mathscr{H}) \\
& \Rightarrow P x=0 \text { as } P \leq I-N_{S} \\
& \Rightarrow x=(I-P) x .
\end{aligned}
$$

Thus we have $N_{S P}=I-P$. Using the polar decomposition of $S P$, there exists a partial isometry $U: P(\mathscr{H}) \rightarrow Q^{\prime}(\mathscr{H})$. Thus $P \sim Q^{\prime}$. From this it follows that $Q \in \mathfrak{I}$.

Consider $(I-Q) S(I-P)$.

Lemma. (a) $(I-Q) S(I-P)(\mathscr{H})=(I-Q)(\mathscr{H})$. (In particular, the range of $(I-Q) S(I-P)$ is closed. $)$

(b) $N_{((I-Q) S(I-P))^{*}}=Q$.

(c) $N_{(I-Q) S(I-P)}=P+N_{S}$.

Proof of Lemma. (a) $\subseteq$ is clear. To obtain the reverse inclusion, let $x \in \mathscr{H}$.

$$
\begin{aligned}
x=(I-Q) x & \Rightarrow Q x=0 \\
& \Rightarrow N_{S^{*}} x=N_{S^{*}} Q x=0 \\
& \Rightarrow x \in \text { range } S \quad \text { (as the range of } S \text { is closed). }
\end{aligned}
$$

Thus

$$
x=S y=S P y+S(I-P) y \quad \text { for some } y \in \mathscr{H} .
$$

Hence

$$
x=(I-Q) x=(I-Q) S P y+(I-Q) S(I-P) y .
$$

But $R_{S P}=Q^{\prime} \leq Q$, so $(I-Q) S P y=0$. Thus $x=(I-Q) S(I-P) y$, and (a) is proved.

(b) This follows from (a).

(c) If $x \in\left(P+N_{S}\right)(\mathscr{H})$, then $x=P x+N_{S} x$. So

$$
\begin{aligned}
(I-Q) S(I-P) x & =(I-Q) S(I-P) P x+(I-Q) S(I-P) N_{S} x \\
& =(I-Q) S(I-P) N_{S} x .
\end{aligned}
$$

But $N_{S} \leq I-P$ so $(I-P) N_{S} x=N_{S} x$, and hence

$$
(I-Q) S(I-P) N_{S} x=(I-Q) S N_{S} x=0 .
$$

Thus $P+N_{S} \leq N_{(I-Q) S(I-P)}$.

To obtain the reverse inequality, let $x \in N_{(I-Q) S(I-P)}(\mathscr{H})$. Let $y=(I-P) x$ and let $y^{\prime}=P x$ so that

$$
0=(I-Q) S(I-P) x=(I-Q) S y .
$$

Thus $S y=Q S y$, which implies $S y \in Q(\mathscr{H})=Q^{\prime}(\mathscr{H})+N_{S^{*}}(\mathscr{H})$. Since $S y \perp$ $N_{S^{*}}(\mathscr{H}), S y \in Q^{\prime}(\mathscr{H})=R_{S P}(\mathscr{H})$. But the range of $S P$ is closed as the range of $S$ is closed and $P \leq I-N_{S}$. Thus $S y=S P z$ for some $z$ and $S(y-P z)=0$. 
This gives that $y \in\left(P+N_{S}\right)(\mathscr{H})$. Hence $x=y+y^{\prime} \in\left(P+N_{S}\right)(\mathscr{H})$. Thus we obtain the desired inequality, and the lemma is proved.

Note that $P \sim Q^{\prime}$ was proven. But we also have

$$
\operatorname{dim}\left(P+N_{S}\right)=\operatorname{dim} P+\operatorname{dim} N_{S}=\operatorname{dim} P^{\prime}+\operatorname{dim} N_{S}=\operatorname{dim} P^{\prime}
$$

since $\operatorname{dim} P^{\prime} \geq \aleph_{0}$ and $\operatorname{dim} P^{\prime} \geq N_{S}$.

Similarly

$$
\operatorname{dim} Q=\operatorname{dim} Q^{\prime}+\operatorname{dim} N_{S^{*}}=\operatorname{dim} P^{\prime}+\operatorname{dim} N_{S^{*}}=\operatorname{dim} P^{\prime} .
$$

Thus $P+N_{S} \sim Q$. To complete the proof of the theorem, choose a partial isometry $W$ with initial space $\left(P+N_{S}\right)(\mathscr{H})$ and range $Q(\mathscr{H})$. Then $\hat{S}=$ $(I-Q) S(I-P)+W$ is invertible and $S-\hat{S} \in \mathfrak{I}$.

As was noted earlier, if $\mathfrak{I}$ is an arbitrary ideal in the von Neumann algebra $\mathfrak{A}$, then there is a unique central projection $P$ such that $P \mathfrak{I}$ is completely noncompact in $P \mathfrak{A}$ and $(I-P) \mathfrak{I}$ is relatively compact in $(I-P) \mathfrak{A}$. We define the index of $T$ to be zero if $i((I-P) T)$ and $\bar{i}(P T)$ are both zero. Combining all the above we have the following theorem.

Theorem 4. Let $\mathfrak{I}$ be an arbitrary ideal in the von Neumann algebra $\mathfrak{A}$, and let $T \in \mathfrak{A}$ be Fredholm with respect to $\mathfrak{I}$. Then

$$
\exists K \in \mathfrak{I} \text { with } T+K \text { invertible } \Leftrightarrow \text { the index of } T \text { is zero. }
$$

Using the fact that the index is continuous on the set of Fredholm elements, see $[3, \S 11]$, we obtain a corollary relating the essential spectrum (relative to $\mathfrak{I}$ ) with the Weyl spectrum (relative to $\mathfrak{I}$ ). First we define these sets.

Definition. Let $\mathfrak{I}$ be an arbitrary ideal in $\mathfrak{A}$, and let $\pi: \mathfrak{A} \rightarrow \mathfrak{A} / \mathfrak{I}$ be the canonical quotient map. For $T \in \mathfrak{A}$ define the essential spectrum of $T$ (relative to $\mathfrak{I})$, denoted by $\sigma_{e}(T)$, to be the set $\sigma(\pi(T))$. Define the Weyl spectrum of $T$ (relative to $\mathfrak{I}$ ), denoted by $\sigma_{w}(T)$, to be the set $\bigcap_{K \in \mathcal{J}} \sigma(T+K)$.

Clearly $\sigma_{e}\left(T \subseteq \sigma_{w}(T)\right.$, but also obtain the following result.

Corollary. For $T \in \mathfrak{A}, \partial\left(\sigma_{w}(T)\right) \subseteq \sigma_{e}(T)$, where $\partial$ denotes the boundary.

Proof. Let $\lambda \in \partial\left(\sigma_{w}(T)\right)$, but suppose $\lambda \notin \sigma_{e}(T)$; i.e., $T-\lambda$ is Fredholm. Choose a sequence $\left\{\lambda_{n}\right\}$ in $\mathbb{C} \backslash \sigma_{w}(T)$ converging to $\lambda$. Since $\lambda_{n} \notin \sigma_{w}(T)$, $\exists K_{n} \in \mathfrak{I}$ with $T+K_{n}-\lambda_{n}$ invertible. Thus $T-\lambda_{n}$ is Fredholm with index zero. By the continuity of the index on the set of Fredholm elements, the index of $T-\lambda$ is zero. Thus by Theorem 4 there is a $K \in \mathscr{F}$ with $T+K-\lambda$ invertible. This contradicts the fact that $\lambda \in \sigma_{w}(T)$.

In the case where $\mathfrak{I}$ is a compact ideal, it was shown that the perturbation can be made as small in norm as we wish. This is not true in general as the following example shows. 
Example 1. Let $\mathscr{H}$ be a separable Hilbert space, let $\mathfrak{A}=\mathscr{B}(\mathscr{H}) \oplus \mathscr{B}(\mathscr{H})$, and let $\mathfrak{I}=0 \oplus \mathscr{B}(\mathscr{H})$. Let $\left\{e_{1}, e_{2}, \ldots\right\}$ be an orthonormal basis for $\mathscr{H}$, and let $S^{*} \in \mathscr{B}(\mathscr{L})$ be the backwards unilateral shift given by

$$
S^{*}\left(e_{j}\right)= \begin{cases}0 & \text { if } j=1, \\ e_{j-1} & \text { if } j>1 .\end{cases}
$$

If $T \in \mathfrak{A}$ is given by

$$
T=\left[\begin{array}{cc}
I & 0 \\
0 & S^{*}
\end{array}\right],
$$

then $T$ is easily seen to be Fredholm with respect to $\mathfrak{I}$. Furthermore, if

$$
K=\left[\begin{array}{cc}
0 & 0 \\
0 & S^{*}-I
\end{array}\right]
$$

then $K \in \mathfrak{I}$ and $T-K=I_{\mathfrak{A}}$. However if

$$
J=\left[\begin{array}{ll}
0 & 0 \\
0 & X
\end{array}\right] \in \mathfrak{I}
$$

is such that $T-J$ is invertible, then $S^{*}-X$ is invertible in $\mathscr{B}(\mathscr{H})$ and so $\|J\| \geq\|X\| \geq 1$.

A similar example shows that the perturbation cannot be made small even in the case where $\mathfrak{A}$ is a factor (over a nonseparable Hilbert space).

Example 2. Let $\mathscr{H}$ be a Hilbert space with $\operatorname{dim} \mathscr{H}>\aleph_{0}$ and let $\mathfrak{A}=\mathscr{B}(\mathscr{H})$. Let $\mathfrak{I}$ be the closed ideal generated by elements of rank $\leq \aleph_{0}$. Defined the operator $T$ as follows: let $P_{1}, P_{2}, \ldots$ be pairwise orthogonal projections with $\operatorname{dim} P_{j}=\aleph_{0}$ and let $P=\sum P_{j}$. Let $U_{j}$ be an isometry from $P_{j}(\mathscr{H})$ onto $P_{j+1}(\mathscr{H})$ and define $T$ by

$$
T=\left[\begin{array}{ccccc}
0 & & & & \\
U_{1} & 0 & & & \\
& U_{2} & \ddots & & \\
& & & \ddots & \\
& & & & I
\end{array}\right]
$$

with respect to the decomposition $\mathscr{H}=\sum P_{j}(\mathscr{C}) \oplus(I-P)(\mathscr{H})$. Note that $T$ is an isometry whose range is $\left(I-P_{1}\right)(\mathscr{H})$. To show that $T$ is Fredholm of index 0 , choose $Q \leq I-P$ such that $\operatorname{dim} Q=\aleph_{0}$ and consider $T(I-Q)$. One can show that $N_{T(I-Q)}=Q$ and range $T(I-Q)=\left(I-\left(P_{1}+Q\right)\right)(\mathscr{H})$. Choose a partial isometry $U$ with initial space $Q(\mathscr{H})$ and range $\left(P_{1}+Q\right)(\mathscr{H})$ so that $T-T Q+U$ is invertible. Suppose, however, that $S$ is invertible and choose $y \in P_{1}(\mathscr{H})$ with $\|y\|=1$. Since $S$ is invertible there is an $x \in \mathscr{H}$ 
with $S x=y$. Thus

$$
\begin{aligned}
\|T-S\|^{2} & \geq \frac{\|T x-S x\|^{2}}{\|x\|^{2}}=\frac{\|T x-y\|^{2}}{\|x\|^{2}} \\
& =\frac{\|T x\|^{2}+\|y\|^{2}}{\|x\|^{2}} \text { as } y \perp T x \\
& =1+\frac{1}{\|x\|^{2}} \\
& >1 .
\end{aligned}
$$

Thus $T$ cannot be perturbed by a small element to obtain an invertible.

\section{REFERENCES}

1. M. Breuer, Fredholm theories in von Neumann algebras I, Math. Ann. 178 (1968), 243-254.

2. V. Kaftal, On the theory of compact operators in von Neumann algebras I, Indiana Univ. Math. J. 26 (1977), 447-457.

3. C. L. Olsen, Index theory in von Neumann algebras, Mem. Amer. Math. Soc., no. 294, Amer. Math. Soc., Providence, RI, 1984.

4. M. Takesaki, Theory of operator algebras. I, Springer-Verlag, New York, 1979.

5. J. Tomiyama, Generalized dimension function for $W^{*}$-algebras of infinite type, Tokoku Math. J. 10 (1958), 121-129.

6. W. Wils, Two-sided ideals in $W^{*}$-algebras, J. Reine Angew. Math. 242-4 (1970), 55-68.

Department of Mathematics and Computer Science, State University of New York, College at Fredonia, Fredonia, New York 14063 